\title{
Significance of Synthetic Cilia and Arrhenius Energy on Double Diffusive Stream of Radiated Hybrid Nanofluid in Microfluidic Pump under Ohmic Heating: An Entropic Analysis
}

\author{
Najma Saleem ${ }^{1, *(D)}$ and Sufian Munawar ${ }^{2}$ (D) \\ 1 Department of Mathematics and Natural Sciences, College of Sciences and Human Studies, \\ Prince Mohammad Bin Fahd University, Khobar 31952, Saudi Arabia \\ 2 Department of Quantitative Methods, College of Business Administration, Imam Abdulrahman \\ Bin Faisal University, P.O. Box 1982, Dammam 34212, Saudi Arabia; sufian.munawar@hotmail.com \\ * Correspondence: nsaleem@pmu.edu.sa
}

check for updates

Citation: Saleem, N.; Munawar, S. Significance of Synthetic Cilia and Arrhenius Energy on Double Diffusive Stream of Radiated Hybrid Nanofluid in Microfluidic Pump under Ohmic Heating: An Entropic Analysis. Coatings 2021, 11, 1292. https://doi.org/10.3390/ coatings11111292

Academic Editors: Rodica Borcia and Rahmat Ellahi

Received: 9 September 2021

Accepted: 21 October 2021

Published: 25 October 2021

Publisher's Note: MDPI stays neutral with regard to jurisdictional claims in published maps and institutional affiliations.

Copyright: (c) 2021 by the authors. Licensee MDPI, Basel, Switzerland. This article is an open access article distributed under the terms and conditions of the Creative Commons Attribution (CC BY) license (https:/ / creativecommons.org/licenses/by/ $4.0 /)$.

\begin{abstract}
This study investigates the thermal aspects of magnetohydrodynamic double diffusive flow of a radiated $\mathrm{Cu}-\mathrm{CuO} / \mathrm{Casson}$ hybrid nano-liquid through a microfluidic pump in the presence of electroosmosis effects. Shared effects of the Arrhenius activation energy and the Joule heating on the intended liquid transport are also incorporated. The inner wall of the pump is covered with electrically charged fabricated cilia mat that facilitates flow actuation and micro-mixing process. The governing equations for the proposed problem are simplified by utilizing the Debye-Hückel and lubrication approximations. The numerical solutions are calculated with the aid of shooting technique. The analysis reports that the substantial effects of electroosmosis contribute an important role in cooling process. Existence of electric double layer stimulates an escalation in liquid stream in the vicinity of the pump surface. The Arrhenius energy input strengthens the mass dispersion and regulates the thermal treatment. The proposed geometry delivers a deep perception that fabricated cilia in electroosmotic pumps are potential pharmaceutical micromixers for an effective flow and minimum entropy generation rate.
\end{abstract}

Keywords: entropy formation; ciliary transport; electric double layer; magnetic field; hybrid nanofluid; Arrhenius energy

\section{Introduction}

Motile cilia are flexible filaments attached to the cell surface and assist in the directional transport of the cell body itself or various substances passing over the surface. The arrays of cilia perform like oars by embracing an elliptic recurring motion and generate a train of propagating waves namely metachronal waves. Motile cilia play a considerable role in the proper functioning of the respiratory tract, digestive system, nervous system, and reproductive systems in males and females [1-5]. Recently, at micro-level artificial ciliacoated appliances such as micro-mimicking actuators, micro-robots, nano-micromotors, and actuators have fascinated many investigators to explore more ideas in the field of artificial intelligence. These micromixers have essential applications in physiological procedures, pharmaceutical industry, bioengineering, artificial intelligence, and regulation of liquid stream. Understanding the significance of cilia stimulated flows in biology and bioengineering disciplines, some interested contributions are given for the relevant audience [6-8].

Electroosmotic pumps (EOP) are essential applications in microfluidic systems that are commonly used in liquid pumping and manipulation. EOP works under electroosmosis phenomenon. Due to cost effectiveness and growing fabrication technologies, EOPs have been extensively used in physiological and engineering fields. An electroosmotic stream evolves with the exertion of electric field on fluid running in microchannels, capillaries, 
membranes, or tubes etc. This induced flow develops a non-neutral coating on the wallliquid boundary line named as electric double layer. The width of this layer performs a decisive role in controlling the electric potential on liquid stream. Models of some advanced electronic apparatus in microfluidic designs are electroosmotic liquid pumps, drug supply pharmaceutical pipes, heat exchangers, lab-on-a-chip basis systems, and micro engineered machines. In this regime, Burgreen and Nakache [9] have presented an innovative attempt. The implication of electroosmotic phenomenon in micro/nanofluidic devices was highlighted by Wang et al. [10]. Chaube et al. [11] have investigated an electrokinetical flow of non-Newtonian fluid and reported its potential applications in lab-on-chip-based machines. Saleem et al. [12] studied the influence of electric field on radiated hybrid nanofluid transport under Debye-Hückel and lubrication approximations. Munawar [13] presented a mathematical model of electro-kinetically propelled stream of biomagnetic fluid through a flexible wavy ciliated surface and derived a conclusion that electroosmosis induces acceleration in liquid flow near the ciliated surface. Munawar and Saleem [14] examined the joint impact of magnetic and electric field on nanofluid stream through a micro channel and reported that the escalated value of electroosmotic parameter generates an enhancement liquid velocity close to the channel boundary. Some modern investigations based on the perception of fluid flow interaction with electric and magnetic fields are cited in [15-17].

Low thermal conductivity is the main limitation in the preparation of energy-carrying heated fluids (for their use in industry and physiology). The conventional liquid with small thermal conductivity acquires enhanced thermal transfer features by attaining elevated thermal conductivity when mix with solid nano-sized granules. Thus, a hybrid style nanofluid is a material which mixes chemical and physical attributes of various constituents in solo homogeneous substance. A groundbreaking investigation to enhance the thermal conduction property of conventional liquids was presented by Choi [18]. Chakraborty and Panigrahi [19] have discussed the applications of nanofluids in micro/nanofluidic pumps. An interesting contribution elaborating the combined impacts of Joule heating and heat radiations on hybrid nanofluid stream in a rotating frame was given by Chamkha et al. [20]. Heat characteristics of $\mathrm{Cu}-\mathrm{CuO}$ nanogranules scattered in viscous fluid and micropolar fluid were examined by Iqbal et al. [21] and Tripathi et al. [22]. Some current reports dispensing with hybrid type nanofluid stream through micropumps are listed in [23-25].

Deterioration in free energy is an interesting trend that is associated with numerous biological phenomena under chemical reactions. For example, chemical reactions in consequence of metabolism generates free energy in living creatures and hence causes a significant amount of entropy formation. Endergonic reactions need input energy and hence the initial state conserves lesser energy as compared to the final state. Therefore, a small quantity of energy input called activation energy is desired to start the process. Owing to these substantial applications of heat transfer in physiology and chemical engineering, several studies [26-30] have been conducted to investigate the thermal aspects of bioliquid flows in different regimes. In this regard, a well-known attempt was submitted by Bejan [31]. Thermal study of non-Newtonian transport through a stretching surface with the provision of activation energy input have been presented by Fwaz et al. [32]. Some interesting studies, revealing the exergy analysis of magnetohydrodynamic fluid transport through stretchable surface were given by Munawar and Saleem [33,34].

Inspired from the above discussion, the core motivation of the intended study is in four aspects:

(i) Examine the entropic features of radiated hybrid type nanofluid transport that will be first time deliberated in electroosmotic pump with its electrically charged surface shielded with fabricated cilia

(ii) Double diffusion effects on $\mathrm{Cu}-\mathrm{CuO} / \mathrm{Casson}$ nanofluid transport with the provision of activation energy input. 
(iii) Further, the entropy is supposed to be caused by the heat transfer due the radiation, Joule heating, mass and heat transfer, and viscous irreversibilities; and

(iv) Establish optimal geometry of microfluidic pump for effective flow and minimum entropy generation rate. To the best of authors' comprehension such study has not been conducted earlier.

\section{Problem Statement}

Consider a two-dimensional mixed convective pumping stream of thermally radiated hybrid nature nanofluid flow obtained by mixing copper $\mathrm{Cu}$ and copper oxide $\mathrm{CuO}$ nanobites in blood modeled as a Casson fluid. The flow is assumed to be passed through a symmetric flexible pump with its inner surface covered with field of cilia. It is further contemplated that the transport is influenced by a Lorentz force $\mathbf{J} \times \mathbf{B}$ generated by a transverse magnetic field $\mathbf{B}=\left(0, B_{0}\right)$ and electric current density $\mathbf{J}=\sigma_{\text {hnf }}(\mathbf{V} \times \mathbf{B})$, where $\sigma_{h n f}$ is the effective electrical conductivity. Further the channel is incorporated by an axially applied electric field $\mathbf{E}=\left(\mathrm{E}_{\mathrm{x}}, 0\right)$. The thermal equilibrium condition holds for both nanoparticles and nanofluid which are mixed thoroughly. For the first-order chemical reaction, the rate constant is assumed to be dependent on the absolute temperature $\bar{T}$ and is provided by the Arrhenius law. The wall temperature $T_{1}$ and concentration $C_{1}$, are supposed to be uniform and kept higher than the ambient temperature and ambient concentration to allow mixed convection. The heat enters or leaves the system by heat conduction as well across the boundaries with per unit heat flux $\mathbf{q}_{r}$. The governing equations for the mixed convection are consequent from the laws of conservation of mass, momentum, and energy and are applied to a continuum with concentration, velocity, and temperature gradients. For an incompressible hybrid nanofluid these governing laws with source terms may be written, respectively, as:

$$
\begin{gathered}
\nabla \cdot \mathbf{V}=0, \\
\rho_{h n f} \frac{D \mathbf{V}}{D t}=-\nabla \bar{P}+\mu_{h n f} \nabla \mathbf{S}+\mathbf{F}-\left[(\rho \beta)_{h n f}\left(\bar{T}-T_{0}\right)-\beta_{c} \rho_{h n f}\left(\bar{C}-C_{0}\right)\right] \mathbf{g} \\
\left(\rho C_{P}\right)_{h n f} \frac{D \bar{T}}{D t}=\nabla \cdot\left(k_{h n f} \nabla \bar{T}\right)+\mathbf{S} \cdot \nabla \mathbf{V}-\nabla \cdot \mathbf{q}_{r}+\mathbf{F} \cdot \mathbf{F}+\left(\rho C_{P}\right)_{h n f}\left[\frac{D_{B}}{T_{0}} \nabla \bar{T} \cdot \nabla \bar{T}+D_{T} \nabla \bar{T} \cdot \nabla \bar{C}\right], \\
\left(\rho C_{P}\right)_{h n f} \frac{D \bar{C}}{D t}=\nabla \cdot\left(D_{B} \nabla \bar{C}\right)+D_{T} \nabla \bar{T} \cdot \nabla \bar{T}-k_{r}{ }^{2}\left(\bar{C}-C_{0}\right) A,
\end{gathered}
$$

where $\mathrm{D} / \mathrm{D} t=\partial / \partial t+\mathbf{V} \cdot \nabla$ is the material derivative, $\bar{P}$ is the fluid pressure, $\mathbf{V}=(\bar{U}, \bar{V})$ the velocity vector, $\bar{T}$ is the temperature, $\bar{C}$ is the concentration, $\mathbf{S}$ is the stress tensor for Casson fluid, $\mathbf{F}=\mathbf{J} \times \mathbf{B}+\rho_{e} \mathbf{E}$ is the net body force comprised of the applied magnetic and electric forces, $\rho_{e}$ is the net charge density, $g$ is the gravity, $\beta_{c}$ is the mass expansion coefficient, $D_{B}$ is the Brownian diffusion coefficient, $D_{T}$ is the thermophoresis diffusion, $k_{r}$ is the pre-exponential factor for the reaction. The rate constant $A$ is assumed to be dependent on the absolute temperature $\bar{T}$ and is provided by the modified Arrhenius equation as

$$
A=\left(\frac{\bar{T}}{T_{0}}\right)^{n} e^{-\frac{E_{r}}{k_{b}}}
$$

where $k_{b}$ is the Boltzmann constant and $E_{r}$ is the activation energy.

The rheological state equation for an isotropic Casson fluid is expressed as [35,36]:

$$
\bar{S}_{i j}=\left\{\begin{array}{cc}
2 e_{i j}\left(\mu_{h n f}+\tau_{0} / \sqrt{2 \pi}\right), & \pi \geq \pi_{c} \\
2 e_{i j}\left(\mu_{h n f}+\tau_{0} / \sqrt{2 \pi_{c}}\right), & \pi<\pi_{c}
\end{array}\right.
$$

where $\tau_{0}$ is the yield stress, $\pi\left(=e_{i j} e_{i j}\right)$ is the product of deformation component $e_{i j}$ with $\pi_{c}$ as its critical value and $\mu_{h n f}$ is the dynamics viscosity of hybrid fluid. 
In Equations (2)-(4), the coefficients $\mu_{h n f} \rho_{h n f},(\rho \beta)_{h n f},\left(\rho C_{p}\right)_{h n f}, \sigma_{h n f}$, and $k_{h n f}$ stand for the nanofluid effective viscosity, the density, the thermal expansion coefficient, the thermal heat capacity, the electrical conductivity, and the thermal conductivity. At the reference temperature of $20^{\circ} \mathrm{C}$ to $30{ }^{\circ} \mathrm{C}$, the thermophysical characteristics of $\mathrm{Cu}-\mathrm{CuO}$ hybrid nanoparticles and the base fluid (blood) can be found in [37] and are declared in Table 1. The mathematical equations elaborating these attributes of hybrid nanofluid, as described in (Ghadikolaei and Gholinia [38]), are stated as:

$$
\begin{gathered}
\mu_{h n f}=\frac{\mu_{f}}{\left(1-\phi_{S 1}\right)^{2.5}\left(1-\phi_{S 2}\right)^{2.5}}, \\
\rho_{h n f}=\rho_{f}\left(1-\phi_{S 1}+\phi_{S 1} \frac{\rho_{S 1}}{\rho_{f}}\right)\left(1-\phi_{S 2}\right)+\phi_{S 2} \rho_{S 2}, \\
(\rho \beta)_{h n f}=(\rho \beta)_{f}\left(1-\phi_{S 2}\right)\left(1-\phi_{S 1}+\phi_{S 1} \frac{(\rho \beta)_{S 1}}{(\rho \beta)_{f}}\right)+\phi_{S 2}(\rho \beta)_{S 2}, \\
\frac{\sigma_{h n f}}{\sigma_{f}}=1+\frac{3 \phi\left(\phi_{S 1} \sigma_{S 1}+\phi_{S 2} \sigma_{S 2}-\phi \sigma_{f}\right)}{\left(\phi_{S 1} \sigma_{S 1}+\phi_{S 2} \sigma_{S 2}+2 \phi \sigma_{f}\right)-\phi \sigma_{f}\left(\phi_{S 1} \sigma_{S 1}+\phi_{S 2} \sigma_{S 2}-\phi \sigma_{f}\right)} \\
\frac{\left(\rho C_{p}\right)_{f}\left(1-\phi_{S 2}\right)\left(1-\phi_{S 1}+\phi_{S 1} \frac{\left(\rho C_{p 1}\right.}{(m n f}\right)+\phi_{S 2}\left(\rho C_{p}\right)_{S 2}}{k_{b f}}=\frac{k_{S 2}+(m-1) k_{b f}-(m-1) \phi_{S 2}\left(k_{b f}-k_{S 2}\right)}{k_{S 2}+(m-1) k_{b f}+\phi_{S 2}\left(k_{b f}-k_{S 2}\right)},
\end{gathered}
$$

with

$$
\frac{k_{b f}}{k_{f}}=\frac{k_{S 1}+(m-1) k_{f}-(m-1) \phi_{S 1}\left(k_{f}-k_{S 1}\right)}{k_{S 1}+(m-1) k_{f}+\phi_{S 1}\left(k_{f}-k_{S 1}\right)}
$$

where $\mu_{\mathrm{f}}, \rho_{f},\left(\rho C_{P}\right)_{f}, \sigma_{\mathrm{f}}$ and $k_{f}$ stand for the viscosity, density, heat capacity, electrical conductivity, and the thermal conductivity of the base fluid, respectively, and $m$ defines the structure of nanoparticles. Particularly, a value of $m=3.7$ corresponds to brick shape, $m=4.9$ relates to cylindrical shape and $m=5.7$ is used for platelets shaped solid particles. The subscripts $S 1$ and $S 2$ are associated with the attributes of $\mathrm{Cu}$ and $\mathrm{CuO}$ solid nanoparticles and the subscript $f$ corresponds to the base fluid (blood). Moreover, $\phi=\phi_{\mathrm{S} 1}+\phi_{\mathrm{S} 2}$, is the total volume fraction of dispersed nanoparticles in the base liquid. The default volume fraction of solid nanoparticles in the current study is assumed to be $2 \%$ of the base fluid (Casson fluid).

Table 1. Thermophysical features of hybrid nanofluid [37].

\begin{tabular}{cccc}
\hline Physical Quantities & Base Liquid & \multicolumn{2}{c}{ Solid Nanoparticles Properties } \\
\hline & blood & $\mathrm{Cu}$ & $\mathrm{CuO}$ \\
$\sigma(1 / \Omega \mathrm{m})$ & $f$ & $S 1$ & $S 2$ \\
$\rho\left(\mathrm{kg} / \mathrm{m}^{3}\right)$ & 0.8 & $59.6 \times 10^{6}$ & $2.7 \times 10^{-8}$ \\
$C_{p}\left(\mathrm{JKg} \mathrm{K}^{-1}\right)$ & 1063 & 8933 & 6320 \\
$K\left(\mathrm{Wm}^{-1} \mathrm{~K}^{-1}\right)$ & 3594 & 385 & 531.8 \\
\hline
\end{tabular}

The flow is induced by the cilia engendering metachronal waves propelling with constant velocity $c$ along the pump wall. These rhythmic waves are driven by power and recovery beats of cilia. The flow is considered in a fixed frame Cartesian coordinate system $(\bar{X}, \bar{Y})$ (see Figure 1). The second frame is the moving frame $(\bar{x}, \bar{y})$ which moves from left 
to right with uniform velocity $c$. The two coordinate systems are interrelated with the following relation:

$$
\bar{x}=\bar{X}-c t, \bar{y}=\bar{Y}, \bar{u}=\bar{U}-c, \bar{v}=\bar{V}
$$

where $(\bar{U}, \bar{V})$ and $(\bar{u}, \bar{v})$ are the velocity vector in fixed and moving frames, respectively.

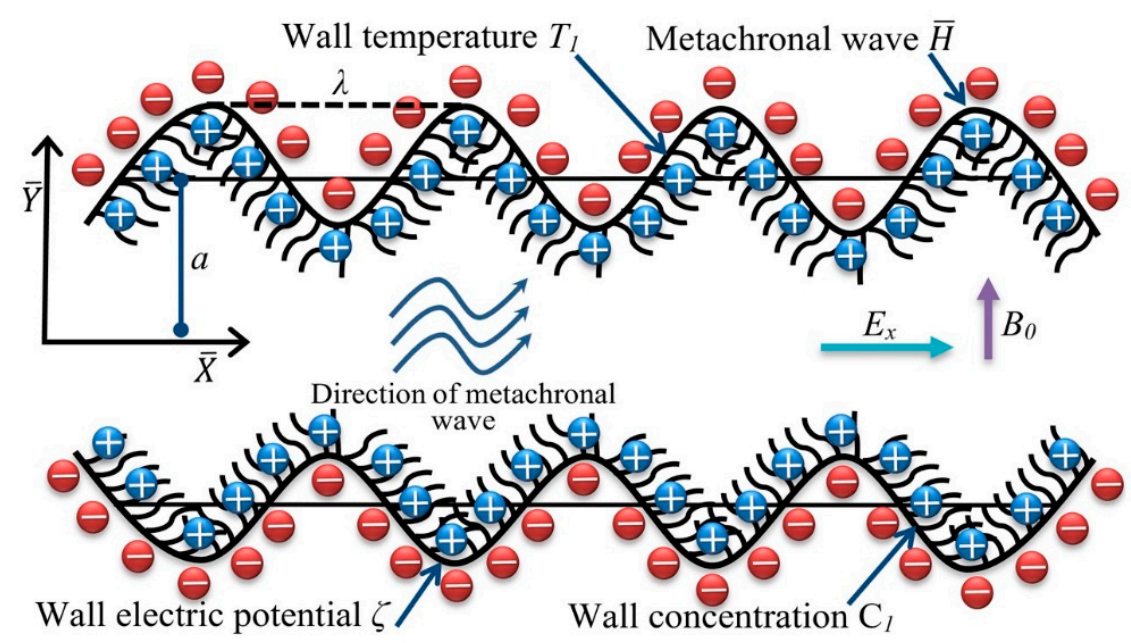

Figure 1. Schematic diagram of the intended flow.

The channel wall surface is described as $[39,40]$ :

$$
f(\bar{X}, t)=\bar{H}=\left[a+a \varepsilon \cos \left(\frac{2 \pi}{\lambda}(\bar{X}-c t)\right)\right] .
$$

The headway of cilia adopts elliptic shaped wave motion and are horizontally located at

$$
g(\bar{X}, t)=X_{0}+a \varepsilon \alpha \sin \left(\frac{2 \pi}{\lambda}(\bar{X}-c t)\right),
$$

where $a$ signifies mean height of the channel, $\alpha$ stands for eccentricity parameter, $\varepsilon$ indicates the length of cilium filament, $\bar{H}$ is the channel's half width, $\lambda$ represents the wavelength of metachronal wave, $t$ is the time parameter, and $X_{0}$ symbolizes the position of the liquid particle.

From Equations (15) and (16), the velocity components at the pump wall can be calculated by using the chain rule as:

$$
\begin{gathered}
\bar{U}_{0}=\left(\frac{\partial g}{\partial t}\right)_{X_{0}}=\frac{-\left(\frac{2 \pi}{\lambda}\right) a c \varepsilon \alpha \cos \left(\frac{2 \pi}{\lambda}(\bar{X}-c t)\right)}{1-\left(\frac{2 \pi}{\lambda}\right) a \varepsilon \alpha \cos \left(\frac{2 \pi}{\lambda}(\bar{X}-c t)\right)}, \\
\overline{V_{0}}=\left(\frac{\partial f}{\partial t}\right)_{X_{0}}=\frac{-\left(\frac{2 \pi}{\lambda}\right) a c \varepsilon \alpha \sin \left(\frac{2 \pi}{\lambda}(\bar{X}-c t)\right)}{1-\left(\frac{2 \pi}{\lambda}\right) a \varepsilon \alpha \sin \left(\frac{2 \pi}{\lambda}(\bar{X}-c t)\right)} .
\end{gathered}
$$

In component form, Equations (1)-(4) in the laboratory frame may be written as:

$$
\frac{\partial \bar{U}}{\partial \bar{X}}+\frac{\partial \bar{V}}{\partial \bar{Y}}=0
$$

$$
\rho_{h n f}\left(\frac{\partial \bar{U}}{\partial t}+\bar{U} \frac{\partial \bar{U}}{\partial \bar{X}}+\bar{V} \frac{\partial \bar{U}}{\partial \bar{Y}}\right)=-\frac{\partial \bar{P}}{\partial \bar{X}}+\frac{\partial \bar{S}_{X X}}{\partial \bar{X}}+\frac{\partial \bar{S}_{X Y}}{\partial \bar{Y}}-\sigma_{h n f} B_{0}^{2} \bar{U}+\rho_{e} E_{x}+g(\rho \beta)_{h n f}\left(\bar{T}-T_{0}\right)+g \beta_{c} \rho_{h n f}\left(\bar{C}-C_{0}\right)
$$




$$
\begin{gathered}
\rho_{h n f}\left(\frac{\partial \bar{V}}{\partial t}+\bar{U} \frac{\partial \bar{V}}{\partial \bar{X}}+\bar{V} \frac{\partial \bar{V}}{\partial \bar{Y}}\right)=-\frac{\partial \bar{P}}{\partial \bar{Y}}+\frac{\partial \bar{S}_{X Y}}{\partial \bar{X}}+\frac{\partial \bar{S}_{Y Y}}{\partial \bar{Y}} \\
\left(\rho C_{P}\right)_{h n f}\left(\frac{\partial \bar{T}}{\partial t}+\bar{U} \frac{\partial \bar{T}}{\partial \bar{X}}+\bar{V} \frac{\partial \bar{T}}{\partial \bar{Y}}\right)=k_{h n f}\left(\frac{\partial^{2} \bar{T}}{\partial \bar{X}^{2}}+\frac{\partial^{2} \bar{T}}{\partial \bar{Y}^{2}}\right)+\bar{S}_{X X} \frac{\partial \bar{U}}{\partial \bar{X}}+\bar{S}_{X Y}\left(\frac{\partial \bar{U}}{\partial \bar{Y}}+\frac{\partial \bar{V}}{\partial \bar{X}}\right)+\bar{S}_{Y Y} \frac{\partial \bar{V}}{\partial \bar{Y}}-\left(\frac{\partial \bar{q}_{r}}{\partial \bar{X}}+\frac{\partial \bar{q}_{r}}{\partial \bar{Y}}\right) \\
+\sigma_{h n f}\left(E_{x}^{2}+B_{0}^{2} \bar{U}^{2}\right)+\left(\rho C_{P}\right)_{h n f}\left[\frac{D_{T}}{T_{0}}\left\{\left(\frac{\partial \bar{T}}{\partial \bar{X}}\right)^{2}+\left(\frac{\partial \bar{T}}{\partial \bar{Y}}\right)^{2}\right\}+D_{B}\left\{\frac{\partial \bar{T}}{\partial \bar{X}} \frac{\partial \bar{C}}{\partial \bar{X}}+\frac{\partial \bar{T}}{\partial \bar{Y}} \frac{\partial \bar{C}}{\partial \bar{Y}}\right\}\right], \\
\left(\frac{\partial \bar{C}}{\partial t}+\bar{U} \frac{\partial \bar{C}}{\partial \bar{X}}+\bar{V} \frac{\partial \bar{C}}{\partial \bar{Y}}\right)=D_{B}\left(\frac{\partial^{2} \bar{C}}{\partial \bar{X}^{2}}+\frac{\partial^{2} \bar{C}}{\partial \bar{Y}^{2}}\right)+\frac{D_{T}}{T_{0}}\left\{\left(\frac{\partial \bar{T}}{\partial \bar{X}}\right)^{2}+\left(\frac{\partial \bar{T}}{\partial \bar{Y}}\right)^{2}\right\}-k_{r}^{2}\left(\frac{\bar{T}}{T_{0}}\right)^{n}\left(\bar{C}-C_{0}\right) e^{-\frac{E_{r}}{k_{r} T}}
\end{gathered}
$$

Introducing the stream function in moving frame

$$
\bar{u}=\frac{\partial \bar{\Psi}}{\partial \bar{y}}, \quad \bar{v}=-\frac{\partial \bar{\Psi}}{\partial \bar{x}} .
$$

The dimensional forms of boundary conditions in moving frame are [35]

$$
\left.\begin{array}{c}
\bar{\Psi}=\bar{F}, \quad \frac{\partial \bar{\Psi}}{\partial \bar{y}}+c=-\frac{\left(\frac{2 \pi}{\lambda}\right) a c \varepsilon \alpha \cos \left(\frac{2 \pi}{\lambda} \bar{x}\right)}{1-\left(\frac{2 \pi}{\lambda}\right) a \varepsilon \alpha \cos \left(\frac{2 \pi}{\lambda} \bar{x}\right)}, \quad \bar{T}=T_{H}, \quad \bar{C}=C_{1} \text { at } \bar{y}=\bar{H}, \\
\bar{\Psi}=0, \quad \frac{\partial^{2} \bar{\Psi}}{\partial \bar{y}^{2}}=0, \quad \frac{\partial \bar{T}}{\partial \bar{y}}=0, \quad \frac{\partial \bar{C}}{\partial \bar{y}}=0 \text { at } \bar{y}=0 .
\end{array}\right\}
$$

In physiological flows, the heat transfer due to radiation is believed to be an important mode of heat transfer. To consider the radiation effect in the channel suppose the radiative heat flux in the flow direction is insignificant in contrast to its magnitude in the normal direction of liquid stream. The radiative heat flux vector $\left(\bar{q}_{r}\right)$ after applying the Rosseland estimation $[41,42]$ is expressed as:

$$
\bar{q}_{r}=-\frac{4 \sigma^{*}}{3 K^{*}} \frac{\partial \bar{T}^{4}}{\partial \bar{Y}},
$$

where $\sigma^{*}$ stands for the Stefan-Boltzmann constant and $K^{*}$ represents the absorption coefficient of nanofluid. The existence of temperature gradient inside the considered stream is supposed to be adequately small. Therefore, the Taylor's series expansion of $T^{4}$ about the temperature gradient, after neglecting the second and higher order terms, gives $\bar{T}^{4} \cong 4\left(T_{1}-T_{0}\right)^{3} \bar{T}-3\left(T_{1}-T_{0}\right)^{4}$. Thus, the radiative heat flux can be written as:

$$
\bar{q}_{r}=-\frac{16 \sigma^{*}\left(T_{1}-T_{0}\right)^{3}}{3 K^{*}} \frac{\partial \bar{T}}{\partial \bar{Y}} .
$$

The Poisson-Boltzmann equation for the electrical potential existing inside the micro pump is described by the following equation as [43]:

$$
\frac{\partial^{2} \bar{\Phi}}{\partial \bar{X}^{2}}+\frac{\partial^{2} \bar{\Phi}}{\partial \bar{Y}^{2}}=-\frac{\rho_{e}}{\epsilon \epsilon_{0}},
$$

where $\epsilon_{0}, \epsilon, \bar{\Phi}$, and $\rho_{e}$ respectively stand for permittivity of free space, permittivity of medium, the electroosmotic potential function, and net charge density. For dual liquid containing positive $\left(\bar{n}_{+}\right)$and counter positive $\left(\bar{n}_{-}\right)$ions with equal charges, the net charge density is identified as:

$$
\rho_{e}=z e\left(\bar{n}_{+}-\bar{n}_{-}\right)=n e^{\frac{-z e \bar{\Phi}}{k_{b} T_{\text {Tave }}}}-n e^{\frac{z e \bar{\Phi}}{k_{b} T_{\text {Tave }}}},
$$


Here $n_{0}$ represents the average intensity of positive and counter positive ions, $z$ is for the valence of ions, $e$ indicates the electric charge, $k_{b}$ indicates the Boltzmann constant, $T_{\text {ave }}$ stands for the local absolute temperature of electrolytic solution. We assume an insignificant concentration gradient inside the liquid and an appropriately low flow Peclet number. This supposition confirms the ionic concentration distribution. On considering the symmetricity of electrolytes, the Boltzmann distribution of net charge density may be quantified as:

$$
\rho_{e}=-2 n z e \sinh \left(\frac{z e \bar{\Phi}}{k_{b} T_{a v e}}\right),
$$

It is considered that wall zeta potential is sufficiently low $(\xi \leq 25 \mathrm{mV})$ and thus the Debyeh-Hückel linearization applies as:

$$
\sinh \left(\frac{z e \bar{\Phi}}{k_{b} T_{a v e}}\right) \cong \frac{z e \bar{\Phi}}{k_{b} T_{a v e}}
$$

Utilizing Equations (29)-(31) in Equation (28), the potential function for electric double layer EDL is found by the equation:

$$
\frac{\partial^{2} \bar{\Phi}}{\partial \bar{X}^{2}}+\frac{\partial^{2} \bar{\Phi}}{\partial \bar{Y}^{2}}=\frac{2 n z^{2} e^{2}}{k_{b} T_{a v e} \epsilon} \bar{\Phi}
$$

At the wall of channel, the electric potential $\bar{\Phi}$ is assumed to be kept at a uniform level of $\zeta$, called zeta-potential.

The following non-dimensional variables are introduced:

$$
\begin{aligned}
& \left.x=\frac{\bar{x}}{\lambda}, \quad y=\frac{\bar{y}}{a}, \quad u=\frac{\bar{u}}{c}, \quad v=\frac{\lambda \bar{v}}{a c}, \quad \Psi=\frac{\bar{\Psi}}{a c}, \quad t=\frac{c \bar{t}}{a}, \quad p=\frac{\bar{p} a^{2}}{\mu_{f} c \lambda}, \quad \Phi=\frac{\bar{\Phi}}{\zeta}, \quad\right\} \\
& \left.\theta=\frac{\bar{T}-T_{0}}{T_{1}-T_{0}}, \quad H=\frac{\bar{H}}{a}, \quad \eta=\frac{\bar{C}-C_{0}}{C_{1}-C_{0}}, \quad \beta=\frac{a}{\lambda}, \quad S=\frac{\bar{s} a}{\mu_{f} c}, \quad \xi=\frac{\mu_{f} \sqrt{2 \pi_{c}}}{\tau_{0}}, \quad F=\frac{\bar{F}}{a c},\right\}
\end{aligned}
$$

where $u$ and $v$ represent dimensionless velocity components along $x$ and $y$ directions, $\beta$ indicates the wave number, $p$ is the nondimensional pressure, $\xi$ is the Casson fluid parameter, $\theta$ stands for the dimensionless temperature field, and $\eta$ denotes the dimensionless mass concentration.

Normalizing the governing Equations (19)-(23) and the EDL Equation (32) using Equations (14) and (33) and applying the approximations of long wavelength and insignificant inertia, one attains the resulting equations of the forms

$$
\begin{gathered}
\frac{\partial p}{\partial x}=\frac{\mu_{h n f}}{\mu_{f}}\left(1+\frac{1}{\xi}\right) \frac{\partial^{3} \Psi}{\partial y^{3}}+\frac{\sigma_{h n f}}{\sigma_{f}} M^{2} \frac{\partial \Psi}{\partial y}+U_{h s} \frac{\partial^{2} \Phi}{\partial y^{2}}+\frac{k_{h n f}}{k_{f}} g_{r} \theta+\frac{\rho_{h n f}}{\rho_{f}} g_{c} \eta, \\
\frac{\partial p}{\partial y}=0,
\end{gathered}
$$

$$
\begin{gathered}
\left(\frac{k_{h n f}}{k_{f}}+R_{n}\right) \frac{\partial^{2} \theta}{\partial y^{2}}+N_{t} \operatorname{Pr}\left(\frac{\partial \theta}{\partial y}\right)^{2}+\frac{\mu_{h n f}}{\mu_{f}} \operatorname{EcPr}\left(1+\frac{1}{\zeta}\right)\left(\frac{\partial^{2} \Psi}{\partial y^{2}}\right)^{2}+\frac{\sigma_{h n f}}{\sigma_{f}} S_{p}+\frac{\sigma_{h n f}}{\sigma_{f}} \operatorname{PrEc} M^{2}\left(\frac{\partial \Psi}{\partial y}\right)^{2}+N_{b} \operatorname{Pr} \frac{\partial \theta}{\partial y} \frac{\partial \eta}{\partial y}=0 \\
\frac{\partial^{2} \eta}{\partial y^{2}}+\frac{N_{t}}{N_{b}} \frac{\partial^{2} \theta}{\partial y^{2}}-\gamma_{1}(1+\tau \theta)^{n} \eta \chi e^{\left(\frac{-E}{1+\tau \theta}\right)}=0 \\
\frac{\partial^{2} \Phi}{\partial y^{2}}=K^{2} \Phi .
\end{gathered}
$$

Eliminating the pressure from Equations (34) and (35) by cross differentiation, one gets:

$$
\frac{\mu_{h n f}}{\mu_{f}}\left(1+\frac{1}{\xi}\right) \frac{\partial^{4} \Psi}{\partial y^{4}}+\frac{\sigma_{h n f}}{\sigma_{f}} M^{2} \frac{\partial^{2} \Psi}{\partial y^{2}}+\frac{k_{h n f}}{k_{f}} g_{r} \frac{\partial \theta}{\partial y}+\frac{\rho_{h n f}}{\rho_{f}} g_{c} \frac{\partial \eta}{\partial y}+U_{\mathrm{hs}} \frac{\partial^{3} \Phi}{\partial y^{3}}=0 .
$$


The dimensionless forms of boundary conditions (25) are given by

$$
\left.\begin{array}{c}
\Psi=0, \frac{\partial^{2} \Psi}{\partial y^{2}}=0, \frac{\partial \Phi}{\partial y}=0, \frac{\partial \theta}{\partial y}=0, \frac{\partial \eta}{\partial y}=0 \text { at } y=0, \\
\Psi=F, \quad \frac{\partial \Psi}{\partial y}=-1-\frac{2 \pi \alpha \varepsilon \beta \cos (2 \pi x)}{1-2 \pi \alpha \varepsilon \beta \cos (2 \pi x)}, \quad \theta=\eta=\Phi=1, \text { at } y=H,
\end{array}\right\}
$$

where $M$ is the Hartmann number, $g_{r}$ the thermal Grashof number, $g_{c}$ the mass Grashof number, Pr the Prandtl number, Ec the Eckert number, $U_{h s}$ Helmholtz-Smoluchowski velocity parameter, K electroosmotic parameter, $R_{n}$ radiation parameter, $S_{p}$ the ohmic heating parameter, $N_{t}$ thermophoresis parameter, $N_{b}$ Brownian motion parameter, $E$ activation energy input parameter for the base liquid, $\chi$ the inverse of thermal heat capacity, and $\gamma_{1}$ is the chemical reaction rate parameter and are expressed respectively as

$$
\left.\begin{array}{c}
M=\sqrt{\frac{\sigma_{f}}{\mu_{f}}} B_{0} a, \quad g_{r}=\frac{g(\rho \beta)_{f} \Delta T a^{2}}{c \mu_{f}}, \quad g_{c}=\frac{g \rho_{f} \beta_{c} \Delta C a^{2}}{c \mu_{f}}, \quad \operatorname{Pr}=\frac{\mu_{f}\left(C_{P}\right)_{f}}{k_{f}}, \quad \text { Ec }=\frac{c^{2}}{\left(C_{P}\right)_{f} \Delta T}, \\
U_{h s}=\frac{-E_{x} \epsilon \epsilon \zeta}{c \mu_{f}}, \quad K=a z e \sqrt{\frac{2 n}{\epsilon \epsilon k_{b} T_{a v e}}}, \quad R_{n}=\frac{16 \sigma^{*}(\Delta T)^{3}}{3 \mu_{f}\left(C_{P}\right)_{f} k_{f}^{*}}, \quad S_{p}=\frac{\sigma_{f} E_{x^{2}}^{2} a^{2}}{\Delta T k_{f}}, \\
N_{t}=\frac{D_{T} \chi \Delta T}{T_{0} v}, \quad N_{b}=\frac{\chi D_{B} \Delta C}{v}, \quad E=\frac{E_{r}}{k_{r} T_{0}}, \quad \chi=\frac{\left(\rho C_{P}\right)_{f}}{\left(\rho C_{P}\right)_{h n f}}, \quad \gamma_{1}=\frac{a^{2} \rho_{f} k_{r}^{2}}{\mu_{f}} .
\end{array}\right\}
$$

The dimensionless mean flow rates for stationary $(Q)$ and moving $(F)$ frames are interrelated as:

$$
F=\int_{0}^{H}\left(\frac{\partial \Psi}{\partial y}\right) d y, \quad Q=1+F
$$

\section{Solution}

The solution of the mixed fourth-order differential Equations (36)-(39) along with the boundary conditions (40) can be obtained by using the built-in numerical package "NDSolve" offered by the computational software Mathematica. While computing the solution the shooting-method utility provided by the software is adopted. The shooting method works by converting an $n$th order ODE to $n$ first-order equations (initial-value problem). The iterative algorithm of shooting method is based on a guess for an unknown (missing) initial condition until the guess meats the boundary condition on the other end. To validate the convergence of present solution scheme, a comparison of present solution is provided with the already published results reported by Akbar and Khan [44] in Figure 2. For comparison the present problem is numerically solved at a limiting case when Buoyancy, mass transfer, and applied electric filed terms were ignored and the exact solution provided in [44] is replotted against the present solution. The figure shows a good agreement between both the velocity profiles. Thus, the comparison establishes the confidence on the present solution technique.

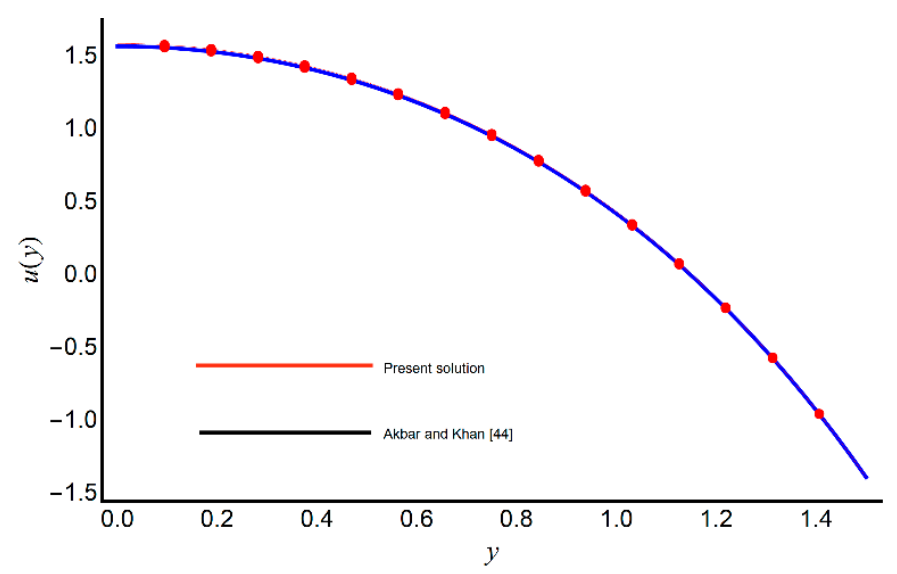

Figure 2. Comparison of velocity profiles by the present numerical scheme and the exact solution reported by [44]. 


\section{Entropy Analysis}

Consider the entropy in the channel is mainly caused by the heat transfer due to convection and radiation, the viscous dissipation, the ohmic heating, and the mass transfer effects. Thus, according to the second law of thermodynamics the entropy expression in moving frame is given as $([45,46])$ :

$$
\begin{aligned}
& S_{\text {gen }}^{\prime \prime \prime}=\frac{1}{T_{0}^{2}}\left[k_{h n f}+\frac{16 \sigma^{*}\left(T_{1}-T_{0}\right)^{3}}{3 K^{*}}\right](\nabla \bar{T})^{2}+\frac{\mu_{h n f}}{T_{0}}\left(1+\frac{1}{\bar{\zeta}}\right)\left(\frac{\partial^{2} \bar{\Psi}}{\partial \bar{y}^{2}}\right)^{2}+\frac{\sigma_{n f}}{T_{0}}\left[B_{0}^{2}\left(\frac{\partial \bar{\Psi}}{\partial \bar{y}}+c\right)^{2}+E_{x}^{2}\right]+\frac{D_{B}}{C_{0}}[\nabla \bar{C} \cdot \nabla \bar{C}]+ \\
& \frac{D_{B}}{T_{0}}[\nabla \bar{T} \cdot \nabla \bar{C}] .
\end{aligned}
$$

Using Equations (14) and (33) in Equation (43), dividing with the characteristic entropy and applying lubrication approximations, one gets the expression for the total entropy generation number as

$$
N_{G}=\underbrace{\left(\frac{k_{h n f}}{k_{f}}+\frac{R_{n}}{\operatorname{Pr}}\right)\left(\frac{\partial \theta}{\partial y}\right)^{2}}_{N_{H}}+\underbrace{\frac{\mu_{h n f}}{\mu_{f}} \frac{\operatorname{PrEc}}{\tau}\left(1+\frac{1}{\xi}\right)\left(\frac{\partial^{2} \Psi}{\partial y^{2}}\right)^{2}}_{N_{F}}+\underbrace{\frac{\sigma_{h n f}}{\sigma_{f} \tau}\left[\operatorname{PrEc} M^{2}\left(\frac{\partial \Psi}{\partial y}+1\right)^{2}+S_{p}\right]}_{N_{J}}+\underbrace{\frac{\omega^{2} \zeta}{\tau^{2}}\left(\frac{\partial \eta}{\partial y}\right)^{2}+\frac{\omega}{\tau L e}\left(\frac{\partial \eta}{\partial y} \frac{\partial \theta}{\partial y}\right)}_{N_{M}},
$$

where $N_{H}$ is the heat transfer, $N_{F}$ the fluid friction, $N_{J}$ is the Joule heating, and $N_{M}$ is the mass transfer irreversibilities, $L e=k_{f} / D_{B} C_{0}$ is the Lewis number, $\omega=\Delta C / C_{0}$ is the dimensionless concentration difference (taken to be 1 ), $\tau=\Delta T / T_{0}$ represents the temperature difference number and is kept equal to 1 . The Bejan number is calculated as:

$$
\mathrm{Be}=\frac{1}{1+\Theta^{\prime}}
$$

where $\Theta$ is the irreversibility ratio and is given by

$$
\Theta=\frac{N_{F}+N_{J}+N_{M}}{N_{H}} .
$$

\section{Results and Discussion}

In this section we exhibit the graphical illustrations and descriptions of numerical solutions for ciliary regulated electroosmotic pumping transport with heat mass transfer in a symmetric channel loaded with hybrid nature Casson nanofluid. The rheological characteristics of the deemed liquid are studied by taking the combination of copper $(\mathrm{Cu})$ and copper oxide $(\mathrm{CuO})$ nano particles with $20 \%$ ratio in base fluid. In Figures 3-10, we have provided visualizations of dynamically or thermodynamically essential quantities for pertinent parameters of interest.

\subsection{Axial Velocity Profile}

The axial velocity profile $u(y)$ of the hybrid nanofluid flow is plotted against $y$ in Figure $3 \mathrm{a}-\mathrm{d}$ to see the effect of the Hartmann number $M$, the Helmholtz-Smoluchowski velocity $U_{h s}$, the thermal Grashof number $g_{r}$, and the solutal Grashof number $g_{c}$. Figure 3a suggests that high values of magnetic field parameter $(M)$ decelerate the fluid velocity in the surroundings of the channel center and boost close to the channel boundary. Moreover, this increase (in Hartmann number) develops a more flatten pattern of velocity profile in the vicinity of the channel center. This diminishing conduct of $M$ on fluid flow is caused by electromotive force which is allied with magnetic force. This stimulated force possesses the ability to repel the fluid stream in the channel deep zone. But in an elastic wavy channel, to sustain a constant flux, an entirely opposite approach can be seen close to the channel walls. Alternations in fluid flow for different values of the Helmholtz-Smoluchowski velocity $U_{h s}$ parameter are reported in Figure $3 \mathrm{~b}$. It is seen that production of $U_{h s}$ velocity in the direction of flow supports the liquid velocity near the edge zone. But the electric field of same intensity, when applied in the reverse direction of the flow induces reduction in liquid 
stream. This drop is more pronounced in the pump center. Figure $3 c$ depicts the impact of thermal Grashof number $\left(g_{r}\right)$ on velocity profile. The thermal Grashof number indicates the comparative impact of buoyancy forces over viscous forces. $g_{r}<1$ suggest the domination of viscous forces over buoyancy forces, whereas $g_{r}>1$ suggests the control of buoyancy forces over viscous forces. As a result of it, a considerable enhancement is associated with liquid velocity profile for exalted values of $g_{r}$ in the contracted pump part and its effects are totally reverse near the channel wall. Figure $3 d$ reveals that solutal Grashof number $\left(g_{c}\right)$ suggests a decline in the vicinity of the pump center and shows inconsequential effects near the wall.

\subsection{Effect of Electroosmosis Parameter}

The profiles of velocity, temperature, concentration, entropy number, and the Bejan number are plotted in Figure 4a-e against electroosmotic parameter $(\mathrm{K})$ at fixed values of the rest of the parameters involved. From Figure $4 a$, it is observed that with an increase in $\mathrm{K}$ the velocity profile in the vicinity of the pump wall weakens and strengthens near the pump center. This conduct is anticipated as an escalation in Debye thickness induces a thin electric double layer and consequently a bulk of liquid flow arises in the pump center. It is also noted that high values of K cools down the liquid temperature (Figure $4 \mathrm{~b}$ ). Figure $4 \mathrm{c}$ suggests electroosmosis assists the mass transfer phenomenon, more considerably near the channel core portion than channel surface. The applied electric field is shown to be an antagonistic impact on entropy formation in the ciliated pump (Figure 4d). Figure 4e demonstrates that the entropy in the channel is dominated by the frictional forces in fluid. This behavior of entropy is owing to the decrease in Debye length which aggregates the electrons concentration and hence suppresses the heat transfer effects in the fluid.

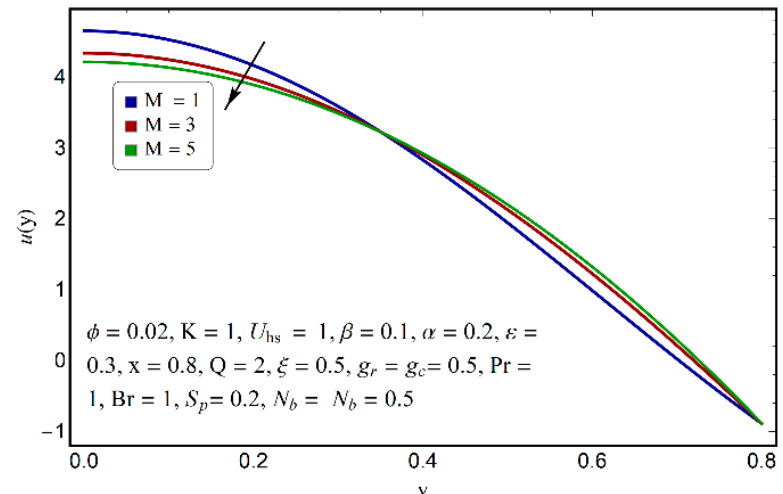

(a)

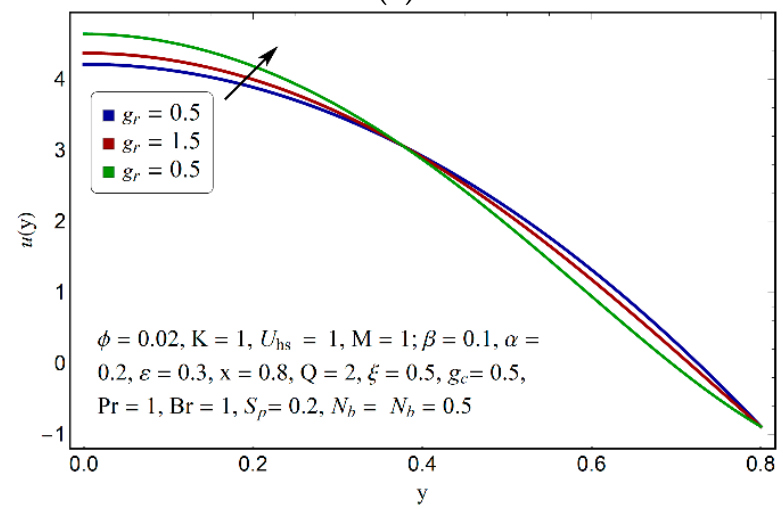

(c)

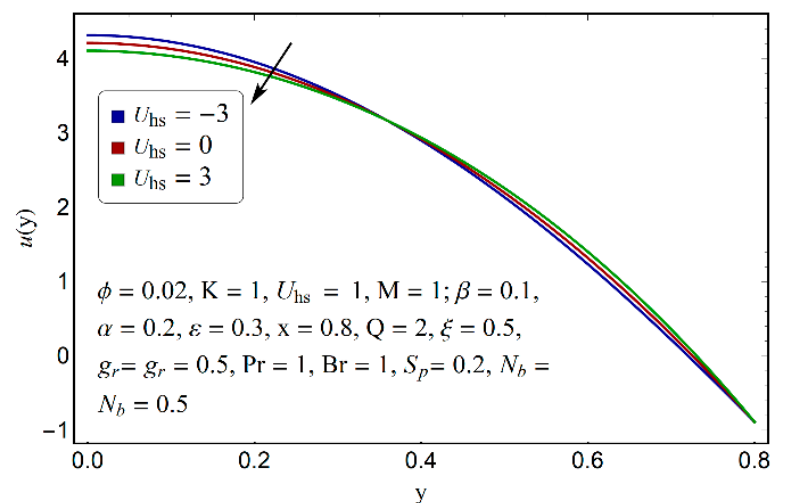

(b)

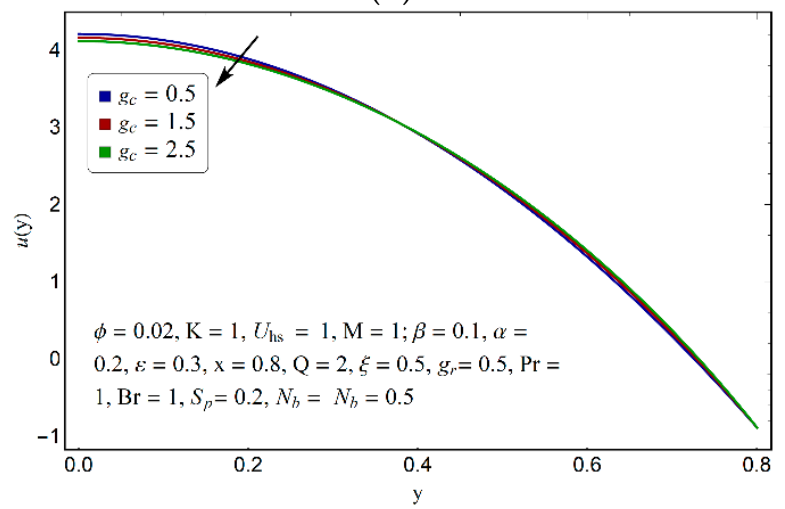

(d)

Figure 3. The axial velocity distribution $u(y)$ versus $y$ for varying (a) magnetic field $\mathrm{M}$ (b) Helmholtz-Smoluchowski velocity $U_{\mathrm{hs}},(\mathbf{c})$ Grashof number $g_{r}$, and (d) solutal Grashof number $g_{c}$. 


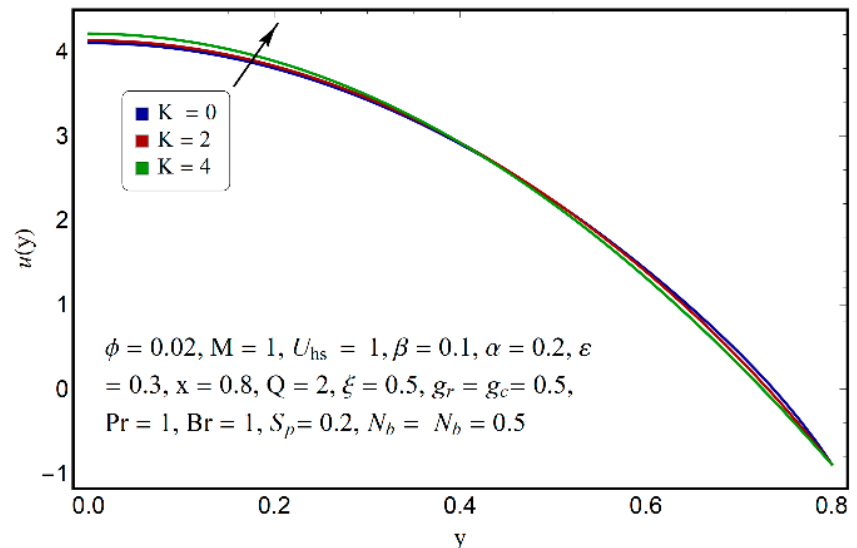

(a)

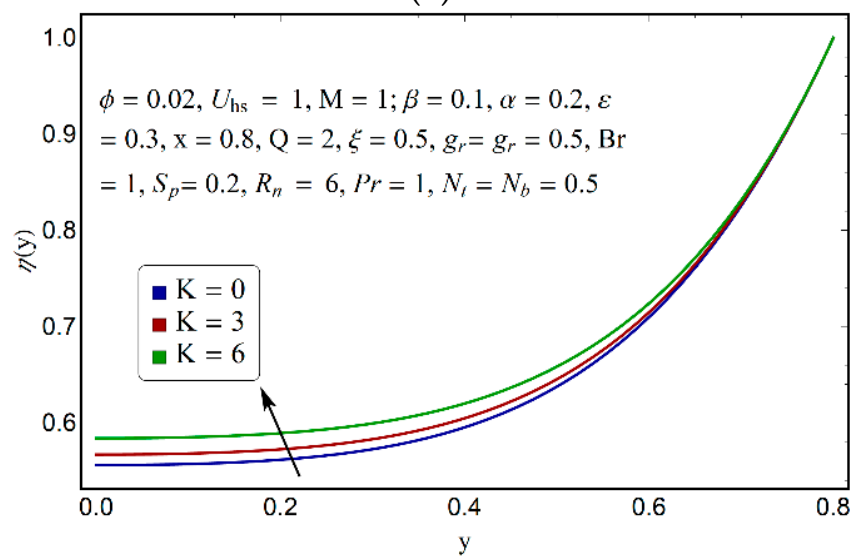

(c)

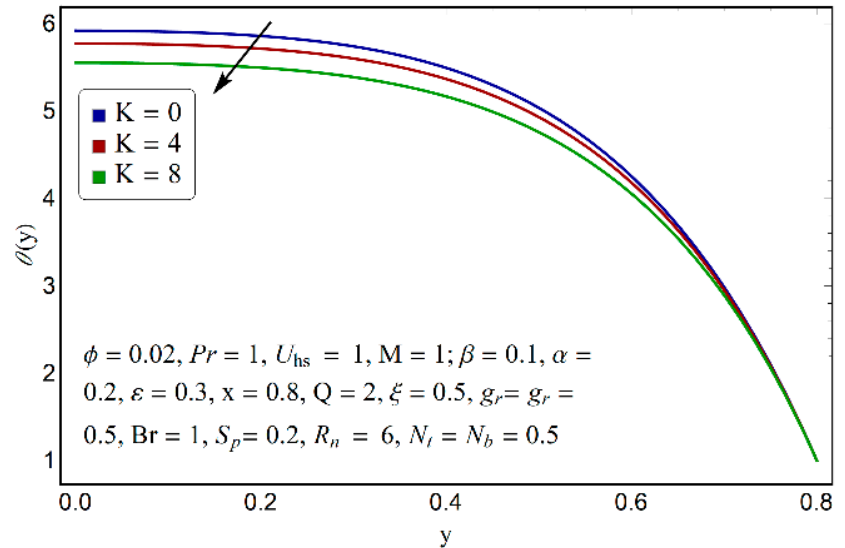

(b)

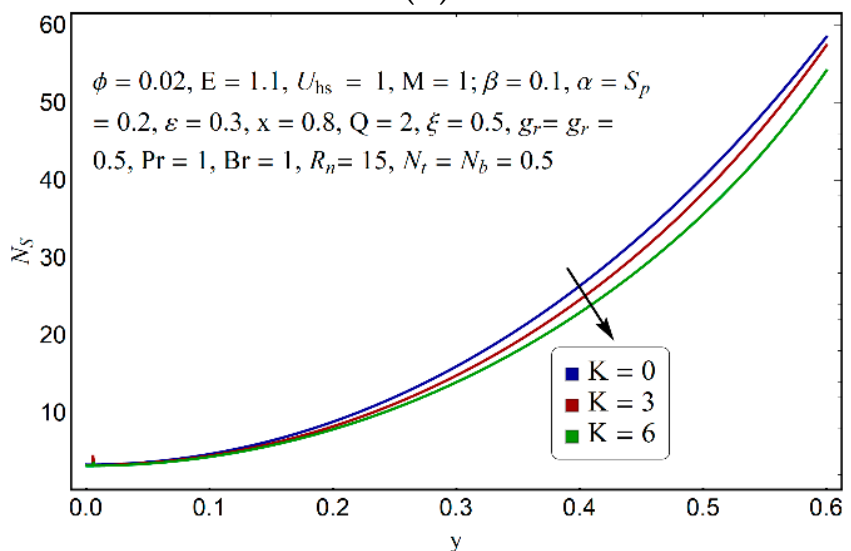

(d)

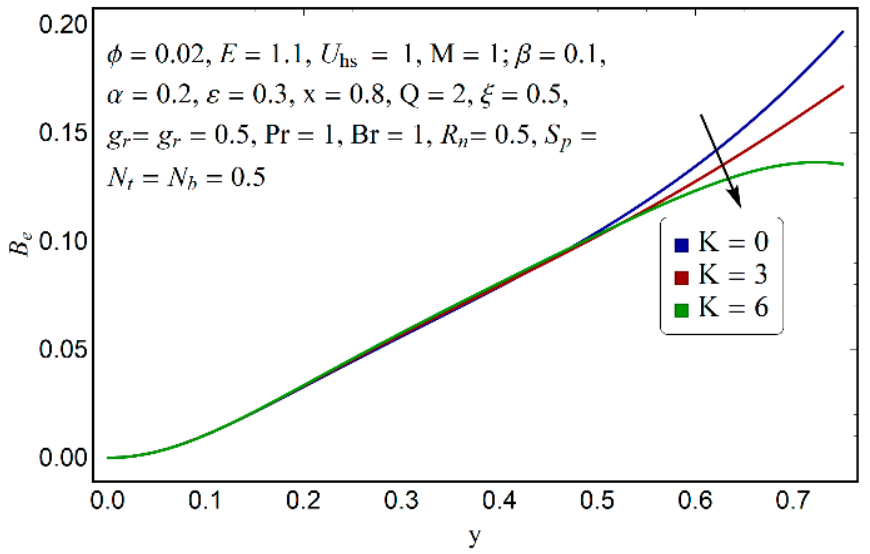

(e)

Figure 4. Impact of $\mathrm{K}$ on (a) the velocity profile, (b) the temperature, (c) the mass concentration, (d) the entropy number, and (e) the Bejan number. 


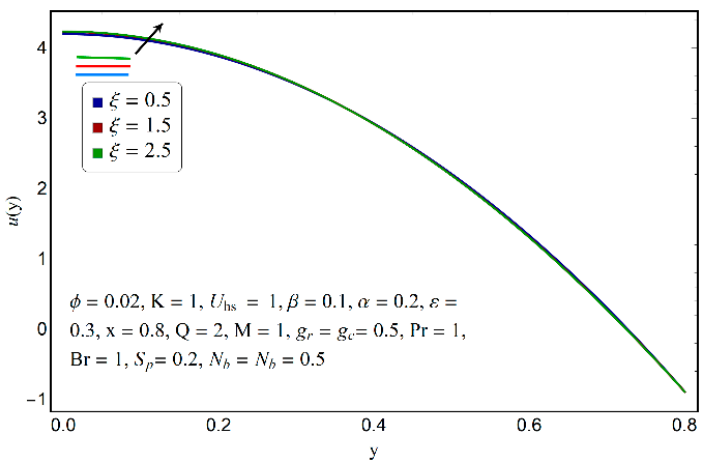

(a)

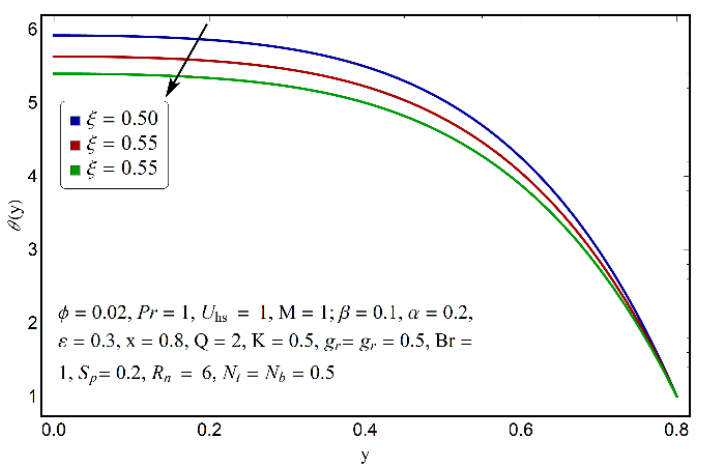

(b)

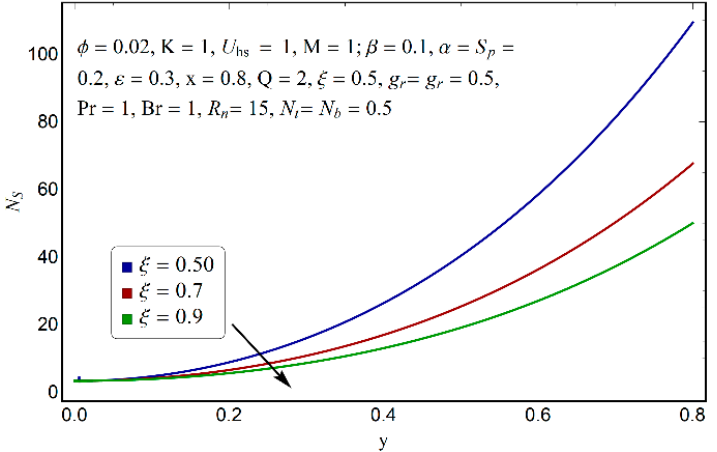

(c)

Figure 5. Impact of $\xi$ on (a) the velocity profile, (b) the temperature profile, and (c) the entropy number.

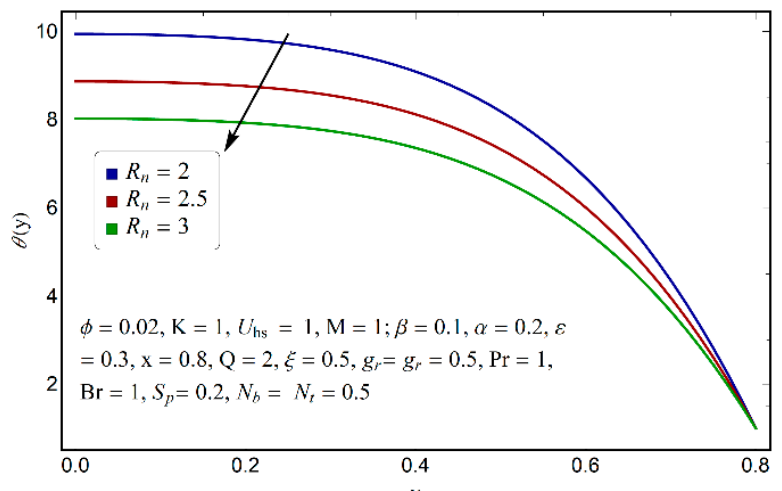

(a)

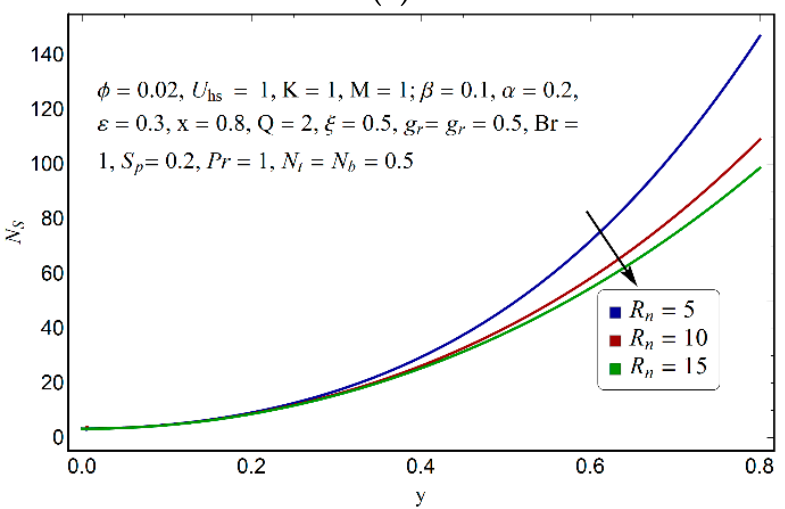

(c)

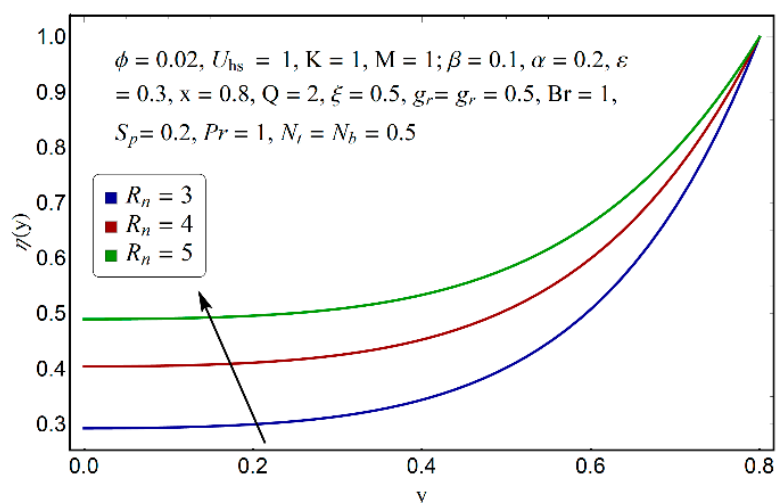

(b)

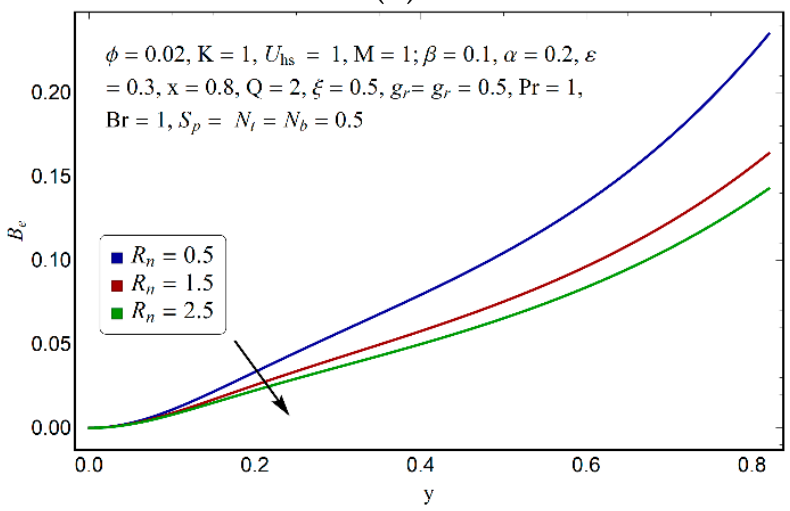

(d)

Figure 6. Impact of $R_{n}$ on (a) the temperature profile, (b) the mass concentration, (c) the entropy number, and (d) the Bejan number. 


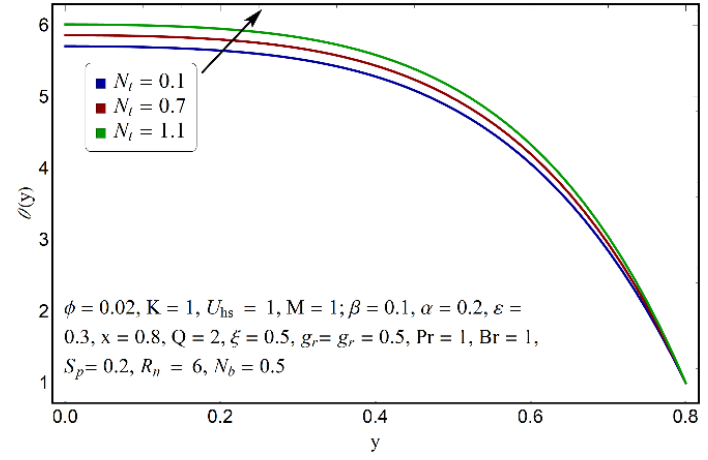

(a)

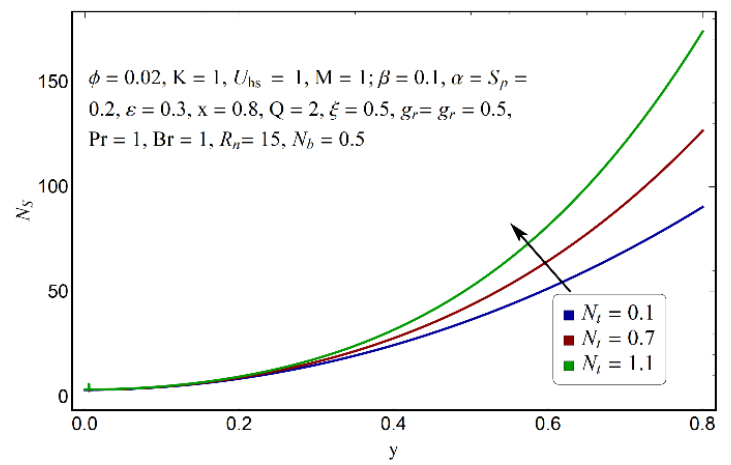

(c)

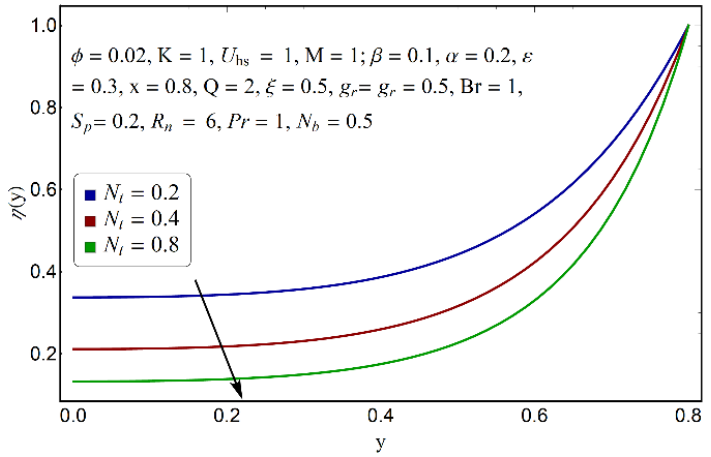

(b)

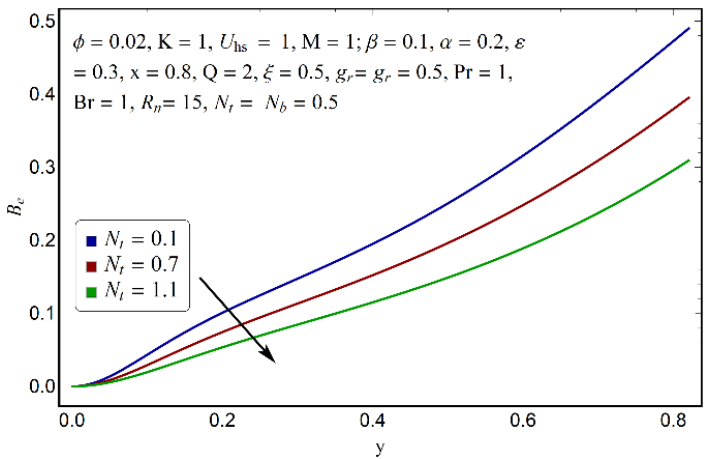

(d)

Figure 7. Impact of $N_{t}$ on (a) the temperature profile, (b) the mass concentration, (c) the entropy number, and (d) the Bejan number.

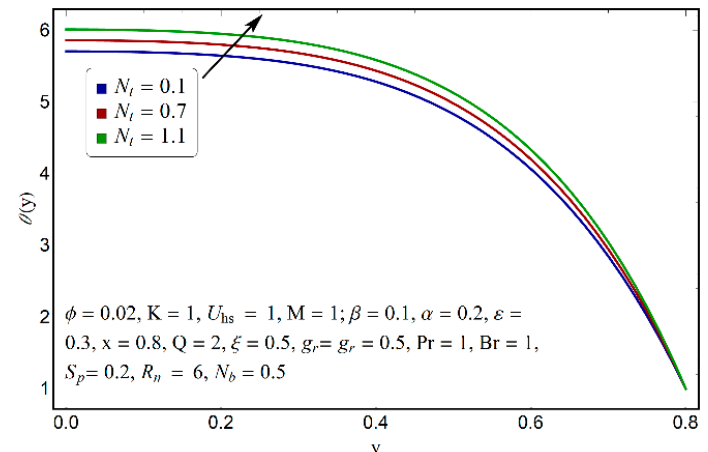

(a)

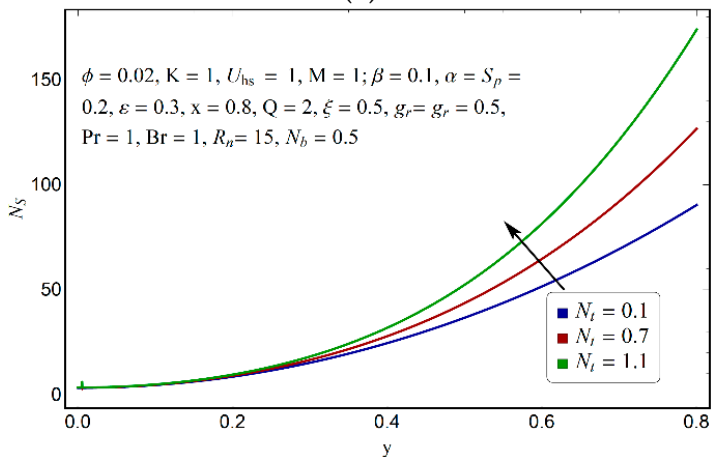

(c)

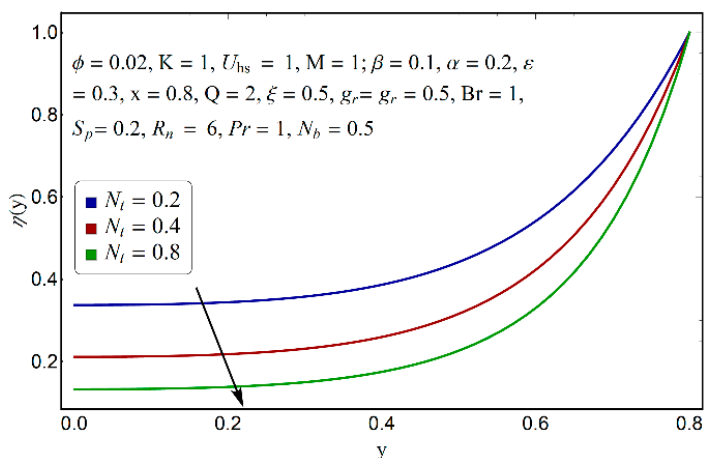

(b)

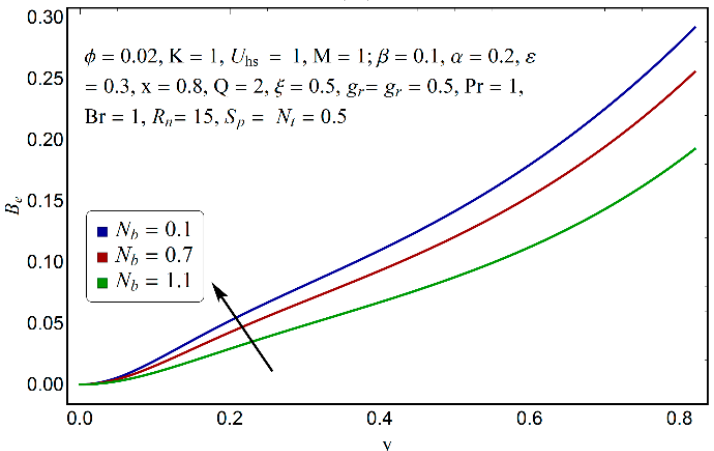

(d)

Figure 8. Impact of $N_{b}$ on (a) the temperature profile, (b) the mass concentration, (c) the entropy number, and (d) the Bejan number. 


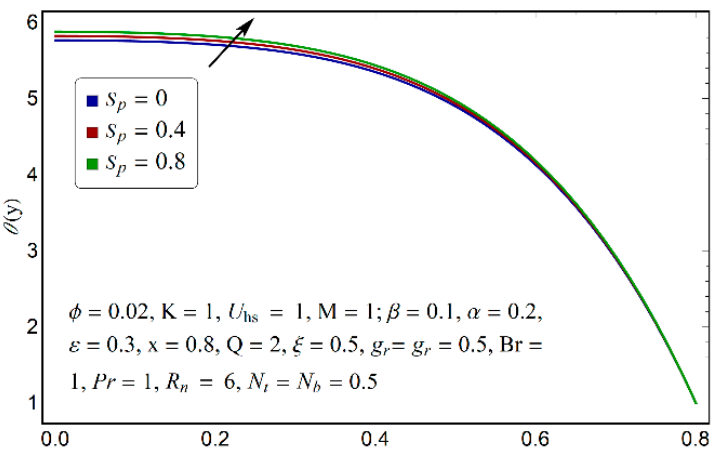

(a)

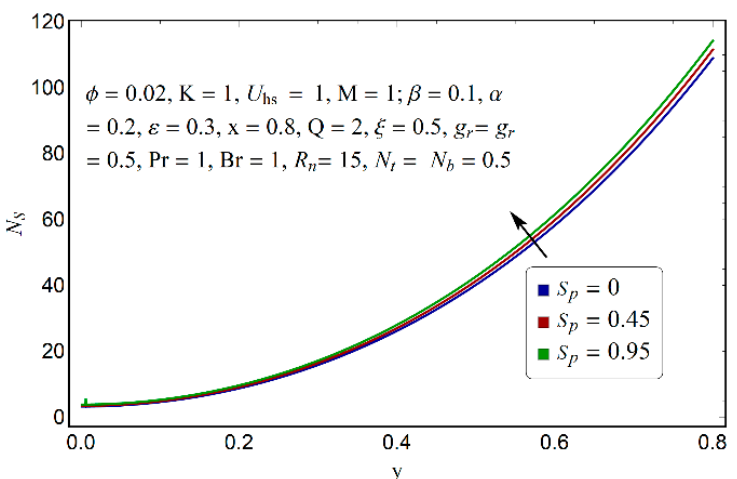

(b)

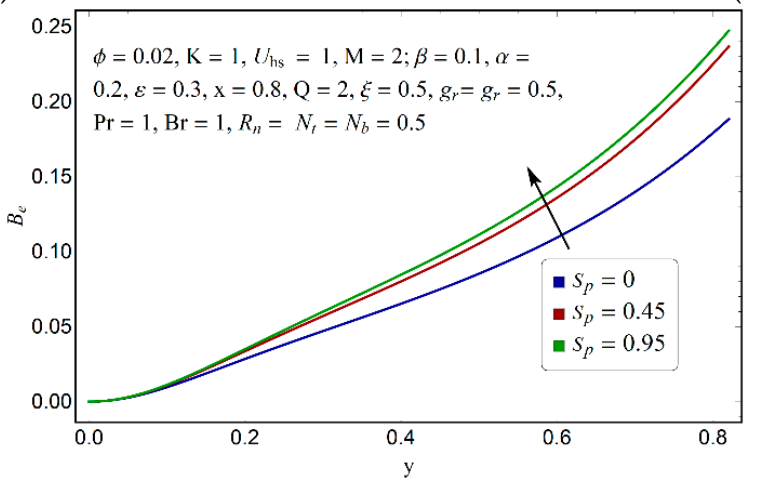

(c)

Figure 9. Impact of $S_{p}$ on (a) the temperature profile, (b) the mass concentration, (c) the entropy number, and (d) the Bejan number.

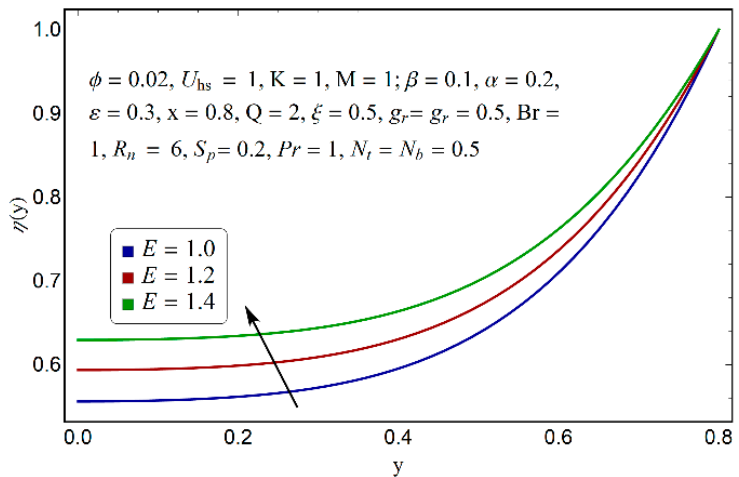

(a)

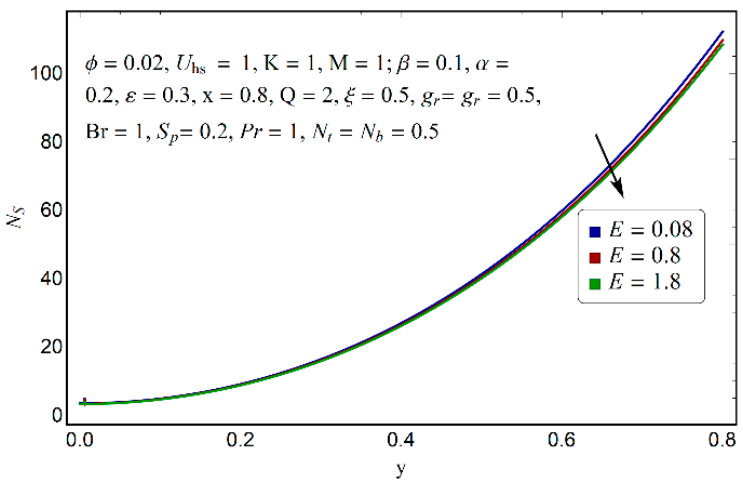

(b)

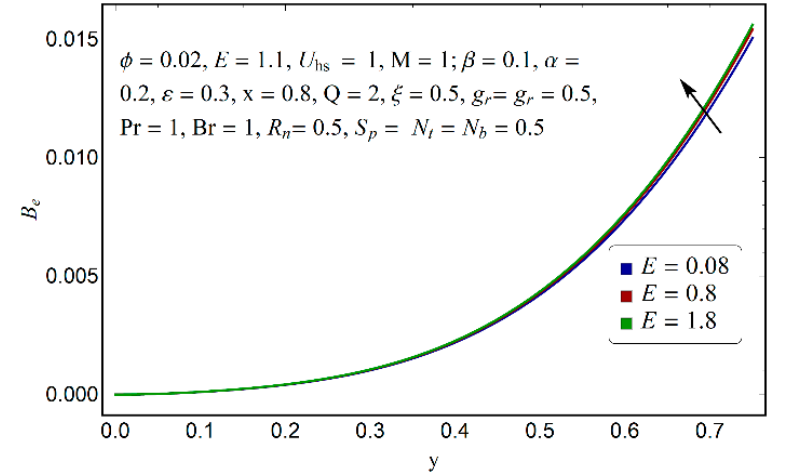

(c)

Figure 10. Effect of varying $E$ on (a) the mass concentration profile, (b) the entropy number, (c) the Bejan number. 


\subsection{Effect of Casson Fluid Parameter}

The impact of Casson fluid material parameter $(\xi)$ on stream velocity, mass concentration, and entropy generation in the channel can be seen through Figure 5a-c. A careful view of Figure 5a reveals that in the channel core part, the Casson fluid flow is more influential than the viscous fluid motion. However, the effect of $\xi$ on fluid velocity is insignificant near the edge part of the channel. Figure $5 b$ is prepared to perceive the impact of $\xi$ on fluid temperature. From this figure, it is concluded that a remarkable drop occurred in fluid temperature for the elevated values of $\xi$. It is also concluded (Figure $5 c$ ) that in order to improve the flow efficiency, higher values of $\xi$ favor the entropy minimization within the channel.

\subsection{Effects Thermal Radiation Number}

Figure $6 \mathrm{a}-\mathrm{d}$ reveals the impact of radiation parameter $R_{n}$ on the temperature profile, concentration distribution, entropy generation number, and the Bejan number. From Figure 6a, a reduction in the temperature profile is noticed for increasing values of $R_{n}$. This conduct is due to the inverse relationship between thermal radiation and heat conduction. An utterly inverse impact of $R_{n}$ on nanoparticles mass concentration is seen in Figure $6 \mathrm{~b}$. The intensifying effects of thermal radiation causes a decay in heat transfer rate inside the pump medium. This drives enough amount of heat far from the system and therefore overall entropy of the system reduces as depicted in Figure 6c. Figure $6 \mathrm{~d}$ illustrates the Bejan number profile against the parameter $R_{n}$. The figure shows that the heat transfer irreversibility weakens as $R_{n}$ increases and contribution of the rest of the irreversibilities becomes stronger. The weakening effect of heat transfer is quite expected since the conduction at the wall decreases for higher values of $R_{n}$.

\subsection{Effects of Thermophoresis Parameter}

Figure 7a portrays the impression of thermophoresis parameter $N_{t}$ on the fluid temperature. By increasing $N_{t}$ the temperature difference rises and thus the phenomenon of thermophoresis augments. The figure shows that the fluid temperature increases as $N_{t}$ increases. This behavior is quite expected since the augmented molecular colloidal motion supports the convection phenomenon and thus strengthens the spread of temperature. Figure $7 \mathrm{~b}$ shows the effect of $N_{t}$ on mass concentration. It is noticed that the mass concentration shows an opposite behavior and decreases as $N_{t}$ increases. The behavior of mass concentration against $N_{t}$ varies and is dependent upon the hybrid solid-particles material. From Figure 7c, it can be viewed that entropy in the channel increases for higher values of $N_{t}$. This is due to the increase in temperature difference within the fluid as thermophoresis phenomenon augments. Figure $7 \mathrm{~d}$ demonstrates that near the channel wall the heat transfer irreversibility dominates the rest of the irreversibilities. Moreover, as the parameter $N_{t}$ increases, the heat transfer irreversibility weakens and other irreversibilities including fluid friction irreversibility become dominant since thermophoresis phenomenon boosts the molecular colloidal and thus the viscous effect.

\subsection{The Effects Brownian Motion Parameters}

Figure 8a shows that the Brownian motion parameter $N_{b}$ triggers fluid temperature due to an increase in the micro-mixing activity. An analogous trend is noticed in mass concentration of nanofluid particles for elevated values of $N_{b}$ (Figure $8 b$ ). It shows that mass concentration is boosted with an increase in $N_{b}$. Figure 8c illustrates that the large values of $N_{b}$ on entropy generation has an escalating outcome. This rise in entropy is owing to the mingling of nanoparticles within the base fluid that boost heat transfer rate. From Figure $8 d$, it can be determined that irreversibility due to fluid friction, ohmic heating, and mass transfer is stronger than heat transfer irreversibility. Moreover, this ascendancy sustains if the elevated values of $N_{b}$ are considered. 


\subsection{Effects of Joule Heating Parameters}

To report the impact of ohmic heating parameter $S_{p}$ on hybrid nanofluid temperature, entropy production in the channel and the Bejan number, Figure $9 \mathrm{a}-\mathrm{c}$, is provided. Figure 9 a states that high values of $S_{p}$ rises fluid temperature near the core region, however its impact on temperature is insignificant near the edge part of the channel. The interaction of electric current with fluid streams induces a conversion of electric energy into thermal energy. This transformation pursues resistive losses and collisions of charged particles. Consequently, the entropy in the channel rises, Figure 9b. From Figure 9c, it can be established that for small values of $S_{p}$ fluid friction and other seem to be more influential than heat transfer irreversibility. However large values of $S_{p}$ can favor irreversibility due to heat transfer.

\subsection{Effects of Activation Energy Parameter}

Figure 10a depicts that the aggregation of nano granules near the pump center seems more frequent compared to the heated wall. Therefore, the mass concentration distribution substantially increases near the pump center for high values of activation energy parameter $E$. It is also viewed in Figure 10b that disorderliness of the system eases with the provision of activation energy input. This conduct may be ascribed to the increasing effect of heat transfer irreversibility over irreversibilities due to other factors for higher values of $E$ (see for instance Figure 10c). It is observed from Figure 10c that the Bejan number slightly increases as $E$ increases, but this variation is insignificant in the center of the channel.

\section{Conclusions}

A mathematical analysis of entropy formation in an electroosmotic ciliated pump loaded with radiated $\mathrm{Cu}-\mathrm{CuO} /$ Casson fluid is performed in this study. Mutual impacts of magnetic field and ohmic heating are also integrated. The stream through the elastic pump is actuated and impelled due to electroosmosis and cilia induced metachronal waves. After employing Debye-Hückel and lubrication approximations, we implement shooting method approach to evaluate the numerical solutions of the intended problem. It is concluded that fluid flow improved near the channel core part for elevated vales of electroosmotic parameter and small values of Hartmann number. Moreover, for the elevated values of electroosmotic parameter the fluid friction irreversibility directs the flow regime. A high electric-potential leads to a reduction in heat transfer effects in the pump. Further, the total entropy inside the channel can be reduced by considering a high electro-kinetically thermally radiated fluid flow. Activation energy input supports fluid particles diffusion but generates heat indulges in the system.

Author Contributions: Conceptualization, N.S.; methodology, N.S.; software, N.S.; validation, N.S.; formal analysis, S.M.; investigation, N.S.; resources, N.S.; writing-original draft preparation, S.M.; writing-review and editing, S.M. and N.S.; visualization, N.S.; supervision, S.M.; project administration, N.S.; funding acquisition, N.S. All authors have read and agreed to the published version of the manuscript.

Funding: This research is supported by the Deanship of Research, Prince Mohammad Bin Fahd University Al-Khobar, Saudi Arabia.

Institutional Review Board Statement: Not applicable.

Informed Consent Statement: Not applicable.

Data Availability Statement: Not applicable.

Conflicts of Interest: The authors declare no conflict of interest. 


\section{References}

1. Knowles, M.R.; Boucher, R.C. Mucus clearance as a primary innate defense mechanism for mammalian airways. J. Clin. Investig. 2002, 109, 571-577. [CrossRef] [PubMed]

2. Kindblom, L.G.; Remotti, H.E.; Aldenborg, F.; Meis-Kindblom, J.M. Gastrointestinal pacemaker cell tumor (GIPACT): Gastrointestinal stromal tumors show phenotypic characteristics of the interstitial cells of Cajal. Am. J. Pathol. 1998, 152, 1259-1269.

3. Wijayagunawardane, M.P.B.; Miyamoto, A. Tumor Necrosis Factor. ALPHA. System in the Bovine Oviduct: A Possible Mechanism for Embryo Transport. J. Reprod. Dev. 2004, 50, 57-62. [CrossRef]

4. Ashraf, M.; Saddiqui, A.M.; Rana, M.A. Fallopian tube assessment of the peristaltic-ciliary flow of a linearly viscous fluid in a finite narrow tube. Appl. Math. Mech. Engl. Ed. 2018, 39, 437-454. [CrossRef]

5. Park, M.S.; Hee, J.J.; Lee, H.J. Roles of Primary Cilia in the Developing Brain. Front. Cell Neurosci. 2019, 13, 218. [CrossRef] [PubMed]

6. Lukens, S.; Yang, X.; Fauci, L. Using Lagrangian coherent structures to analyze fluid mixing by cilia. Chaos 2010, $20,017511$. [CrossRef] [PubMed]

7. Saleem, N.; Munawar, S. Entropy analysis in cilia driven pumping flow of hyperbolic tangent fluid with magnetic field effects. Fluid Dyn. Res. 2020, 52, 025503. [CrossRef]

8. Farooq, A.A.; Siddiqui, A.M. Mathematical model for the ciliary-induced transport of seminal liquids through the ductuli efferentes. Int. J. Biomath. 2017, 10, 1750031. [CrossRef]

9. Burgreen, D.; Nakache, F.R. Electrokinetic Flow in Ultrafine Capillary Slits1. J. Phys. Chem. 1964, 68, 1084-1091. [CrossRef]

10. Wang, X.; Cheng, C.; Wang, S.; Liu, S. Electroosmotic pumps and their applications in microfluidic systems. Microfluid. Nanofluidics 2009, 6, 145-162. [CrossRef] [PubMed]

11. Chaube, M.K.; Yadav, A.; Tripathi, D.; Bég, O.A. Electroosmotic flow of biorheological micropolar fluids through microfluidic channels. Korea-Aust. Rheol. J. 2018, 30, 89-98. [CrossRef]

12. Saleem, N.; Munawar, S.; Tripathi, D. Thermal analysis of double diffusive electrokinetic thermally radiated TiO2-Ag/blood stream triggered by synthetic cilia under buoyancy forces and activation energy. Phys. Scr. 2021, 96, 095218. [CrossRef]

13. Munawar, S. Significance of Slippage and Electric Field in Mucociliary Transport of Biomagnetic Fluid. Lubricants 2021, 9, 48. [CrossRef]

14. Munawar, S.; Saleem, N. Entropy generation in thermally radiated hybrid nanofluid through an electroosmotic pump with ohmic heating: Case of synthetic cilia regulated stream. Sci. Prog. 2021, 104, 00368504211025921. [CrossRef]

15. Yang, C.; Li, D. Electrokinetic Effects on Pressure-Driven Liquid Flows in Rectangular Microchannels. J. Colloid Interface Sci. 1997, 194, 95-107. [CrossRef]

16. Hang, H.U.; Pop, I.; Sun, Q. Fluid flow driven along microchannel by its upper stretching wall with electrokinetic effects. Appl. Math. Mech. Engl. Ed. 2018, 39, 395-408.

17. Akram, J.; Akbar, N.S.; Tripathi, D. Numerical study of the electroosmotic flow of Al2O3-CH3OH Sisko nanofluid through a tapered microchannel in a porous environment. Appl. Nanosci. 2020, 10, 4161-4176. [CrossRef]

18. Choi, S.U.S. Enhancing thermal conductivity of fluids with nanoparticles. In Developments and Applications of Non-Newtonian Flows; ASME: New York, NY, USA, 1995; Volume 66, pp. 99-105.

19. Chakraborty, S.; Panigrahi, P.K. Stability of nanofluid: A review. Appl. Therm. Eng. 2020, 174, 115259. [CrossRef]

20. Chamkha, A.J.; Dogonchi, A.S.; Ganji, D.D. Magneto-hydrodynamic flow and heat transfer of a hybrid nanofluid in a rotating system among two surfaces in the presence of thermal radiation and Joule heating. AIP Adv. 2019, 9, 025103. [CrossRef]

21. Iqbal, Z.; Akbar, N.S.; Azhar, E.; Maraj, E.N. Performance of hybrid nanofluid (Cu-CuO/water) on MHD rotating transport in oscillating vertical channel inspired by Hall current and thermal radiation. Alex. Eng. J. 2018, 57, 1943-1954. [CrossRef]

22. Tripathi, D.; Parkash, J.; Tiwari, A.K.; Ellahi, R. Thermal, microrotation, electromagnetic field and nanoparticle shape effects on $\mathrm{Cu}-\mathrm{CuO} /$ blood flow in microvascular vessels. Microvasc. Res. 2020, 132, 104065. [CrossRef] [PubMed]

23. Mekheimer, K.; Abo-Elkhair, R.; Moawad, A. Electrothermal transport via gold nanoparticles as antimicrobials of blood flow through an electro-osmosis artery with overlapping stenosis. Int. J. Fluid Mech. Res. 2020, 47, 135-152. [CrossRef]

24. Munawar, S.; Saleem, N.; Chamkha, A.J.; Mehmood, A.; Dar, A. Lubricating hot stretching membrane with a thin hybrid nanofluid squeezed film under oscillatory compression. Eur. Phys. J. Plus. 2021, 136, 1-18.

25. Akram, S.; Zafar, M.; Nadeem, S. Peristaltic transport of a Jeffrey fluid with double-diffusive convection in nanofluids in the presence of inclined magnetic field. Int. J. Geom. Methods Mod. Phys. 2018, 15, 1850181. [CrossRef]

26. Farooq, S.; Khan, M.I.; Waqas, M.; Hayat, T.; Alsaedi, A. Transport of hybrid type nanomaterials in peristaltic activity of viscous fluid considering nonlinear radiation, entropy optimization and slip effects. Comput. Methods Programs Biomed. 2020, 184, 105086. [CrossRef] [PubMed]

27. Mehmood, A.; Iqbal, M.; Khan, S.; Munawar, S. Entropy analysis in moving wavy surface boundary-layer. Therm. Sci. 2019, 23, 233-241. [CrossRef]

28. Balla, H.H.; Abdullah, S.; Mohdfaizal, W.; Zulkifli, R.; Sopian, K. Numerical study of the enhancement of heat transfer for hybrid CuO-Cu nanofluids flowing in a circular pipe. J. Oleo. Sci. 2013, 62, 533-539. [CrossRef]

29. Khan, A.A.; Masood, F.; Ellahi, R.; Bhatti, M.M. Mass transport on chemicalized fourth-grade fluid propagating peristaltically through a curved channel with magnetic effects. J. Mol. Liq. 2018, 258, 186-195. [CrossRef] 
30. Hayat, T.; Nisar, Z.; Alsaedi, A.; Ahmed, B. Analysis of activation energy and entropy generation in mixed convective peristaltic transport of Sutterby nanofluid. J. Therm. Anal. Calorim. 2021, 143, 1867-1880. [CrossRef]

31. Bejan, A. Second-law analysis in heat transfer and thermal design. In Advances in Heat Transfer; Hartnett, J.P., Irvine, T.F., Eds.; Elsevier: Amsterdam, The Netherlands, 1982; Volume 15, pp. 1-58.

32. Alsaadi, F.; Ullah, I.; Hayat, T.; Alsaedi, F.E. Entropy generation in nonlinear mixed convective flow of nanofluid in porous space influenced by Arrhenius activation energy and thermal radiation. J. Therm. Anal. Calorim. 2020, 140, 799-809. [CrossRef]

33. Munawar, S.; Saleem, N. Second Law Analysis of Ciliary Pumping Transport in an Inclined Channel Coated with Carreau Fluid under a Magnetic Field. Coatings 2020, 10, 240. [CrossRef]

34. Munawar, S.; Saleem, N. Thermal analysis of an Eyring-Powell fluid flow-through a constricted channel. Therm. Sci. 2020, 24, 1207-1216. [CrossRef]

35. Makinde, O.D.; Reddy, M.G. MHD peristaltic slip flow of Casson fluid and heat transfer in channel filled with a porous medium. Trans. Mech. Eng. B 2019, 26, 2342-2355. [CrossRef]

36. Mernone, A.; Mazumdar, J.; Lucas, S. A mathematical study of peristaltic transport of a casson fluid. Math. Comput. Model. 2002, 35, 895-912. [CrossRef]

37. Alghamdi, W.; Alsubie, A.; Kumam, P.; Saeed, A.; Gul, T. MHD hybrid nanofluid flow comprising the medication through a blood artery. Sci. Rep. 2021, 11, 11621.

38. Ghadikolaei, S.; Gholinia, M. 3D mixed convection MHD flow of GO-MoS hybrid nanoparticles in $\mathrm{H}_{2} \mathrm{O}-\left(\mathrm{CH}_{2} \mathrm{OH}\right)_{2}$ hybrid base fluid under the effect of $\mathrm{H}_{2}$ bond. Int. Commun. Heat Mass Transf. 2020, 110, 104371. [CrossRef]

39. Lardner, T.J.; Shack, W.J. Cilia transport. Bull. Math. Biophys. 1972, 34, 325-335. [CrossRef]

40. Munawar, S.; Saleem, N. Entropy Analysis of an MHD Synthetic Cilia Assisted Transport in a Microchannel Enclosure with Velocity and Thermal Slippage Effects. Coatings 2020, 10, 414. [CrossRef]

41. Saleem, N.; Munawar, S.; Mehmood, A.; Daqqa, I. Entropy production in electroosmotic cilia facilitated stream of thermally radiated nanofluid with Ohmic heating. Micromachines 2021, 12, 1004. [CrossRef]

42. Hayat, T.; Rani, S.; Alsaedi, A.; Rafiq, M. Radiative peristaltic flow of magneto nanofluid in a porous channel with thermal radiation. Results Phys. 2017, 7, 3396-3407. [CrossRef]

43. Mekheimer, K.; Zaher, A.Z.; Hasona, W.M. Entropy of AC electro-kinetics for blood mediated gold or copper nanoparticles as a drug agent for thermotherapy of oncology. Chin. J. Phys. 2020, 65, 123-138. [CrossRef]

44. Akbar, N.S.; Khan, Z. Metachronal beating of cilia under the influence of Casson fluid and magnetic field. J. Magn. Magn. Mater. 2015, 378, 320-326. [CrossRef]

45. Munawar, S.; Saleem, N.; Aboura, K. Second law analysis in the peristaltic flow of variable viscosity fluid. Int. J. Exergy 2016, $20,170-185$.

46. Huang, Y.Y.; Zhang, L.J.; Yang, G.; Wu, J.Y. Secondary Flow and Entropy Generation of Laminar Mixed Convection in the Entrance Region of a Horizontal Square Duct. J. Heat Transf. 2017, 140, 034503. [CrossRef] 